\title{
REVIEW
}

\section{A Low Calcium Condition; Important Roles of Ionized Calcium in Biology}

\author{
Hisao Yamaguchi \\ Department of Pathology, School of Medicine, Keio University, Tokyo, Japan
}

(Received for publication on November 8, 1993)

\begin{abstract}
Calcium is the most widely distributed and inevitably important bivalent ion which has an intimate relation both to functions and structures in living organisms. However, studies on the structural and functional alterations induced under a low calcium condition have been ignored. In the last two decades, to clarify the role of ionized calcium in biology, the morpho-functional alterations in various organs and tissues under a low calcium condition provoked by calcium chelating drugs have been examined. In this paper, the range of results obtained through our experiments will be reviewed and the almost miraculous roles of the calcium ion in biology will be discussed. Most of the presented results offer just the beginning of a new era of the research in this field, therefore further continuous studies can be expected. (Keio I Med 43 (2): $63-74$, June 1994)
\end{abstract}

Key words: mesangiolysis, angiolysis, pulmonary hypertension, Bartter's syndrome, renal lesions

Modern pathology was based on the Zellulare Pathologie by Virchow and the basic way of thinking is that the cell or group of cells play an important and inevitable role in all the physico-pathological phenomena. This philosophy is the basis of thinking in pathology even nowadays.

The site where the cells display their functions is the so-called matrix and they preserved the medium for all the cellular functions, therefore biological phenomena should be induced as integrated phenomena between the cellular functions and the biochemical properties of the matrix. Generally speaking, the matrix around the cells is morphologically homogeneous in character and it is difficult to estimate its morphological alteration, so the morphological studies of the matrix have been ignored.

Following this hypothetical thinking, I have encountered an umbilical cord with inflammation where there was no vasa vasorum in the vessels, around which the main component is a loose matrical material called Whalton jelly. Leucocytes which had immigrated into the perivascular matrix remained concentric acellular halo around the vessels (Fig 1). If the mobility of leucocytes is regarded as constant, this morphology is a reflection of the matrical character in situ. Most studies on cellular mobility deal entirely with the cell itself or with effectors, such as chemical mediators or migration inhibition factors ${ }^{1-5}$ that act upon the cell. However, the end result of cellular mobility does not only depend on the activity of the motile cell itself, but also upon the matrix through which mobility is displayed. Since a biologic reaction that involves mobility of cells may also depend on the rheologic characteristics of the matrix, we have attempted ${ }^{6-8}$ to clarify the relationship of leucocyte reaction patterns to experimental agar matrices with "Agar Bullet" method originated by us.

The matrix was characterized numerically as to hardness, brittleness, cohesiveness, elasticity and viscosity using a rheolometer. ${ }^{9,10}$ It was found that among these parameters only the viscosity was a significant factor, with a critical cut-off value of $1000 \times 10^{4} \mathrm{dyne} / \mathrm{sec} / \mathrm{cm}^{2}$ (Figs 2,3). This viscosity is identical to the so-called anchor requirement.

Of course, the conditions obtained with the bactagar experimental model do not necessarily reflect the situation in living tissue, yet the data may help in understanding some of the mechanisms that regulate inflammation and tumor cell infiltration. This method makes it possible to study cellular mobility without complications arising from vascular, neural and fibrous elements that may affect in vivo behaviours. However, 

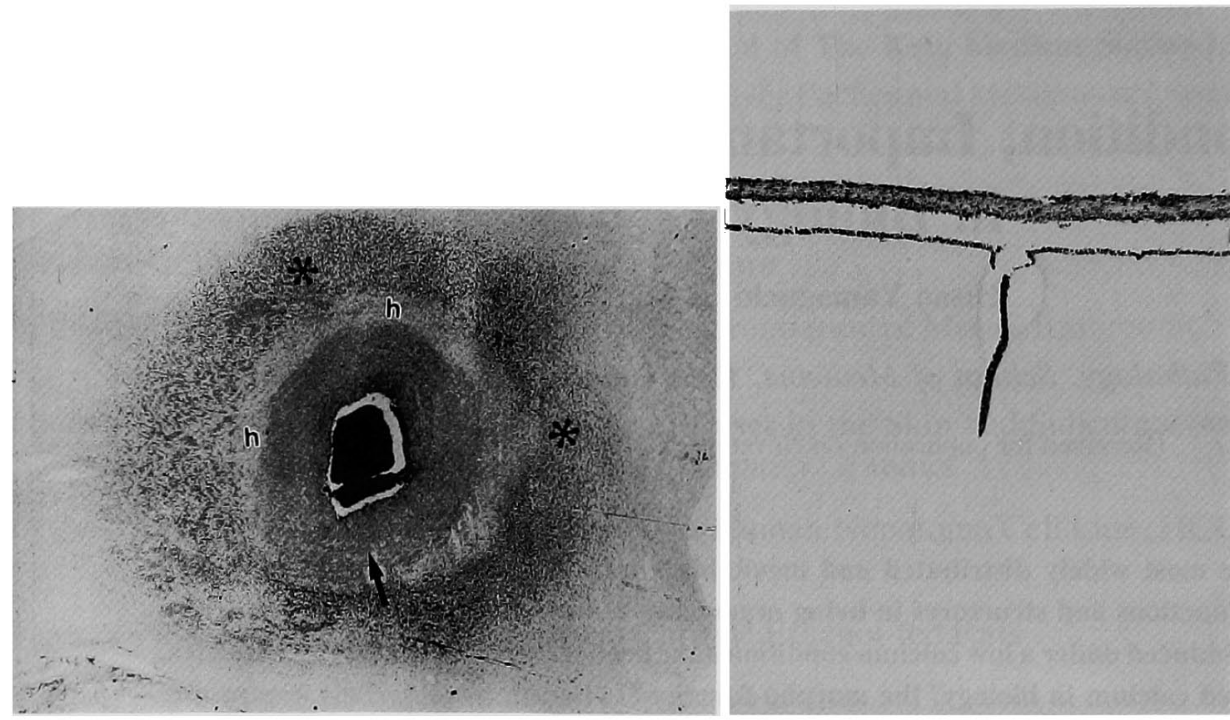

Fig 1 Vasculature in umbilical cord(arrow) in inflammation. Leucocyte reaction zone $\left(^{*}\right)$ is concentrically demonstrated, remaining acellular halo(h) with timelapse. This phemomenon is able to explain that when cell motility would be constant, it depends on the colloid chemical properties of Whalton's jelly whose main component is GAGs $(\mathrm{HE}, \times 20)$.
Fig 2 Cell mobility examined using agar-bullet Method originated by us. Cellular invasion is not seen into $0.7 \%$ agar $(\mathrm{HE}, \times 80)$.

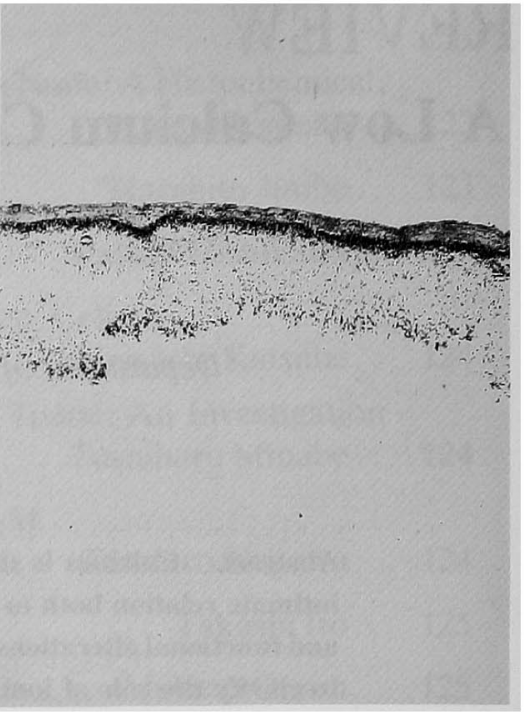

Fig 3 Cellular invasion is observed deeper into agar of $0.62 \%$ in concentration. Viscosity of this agar is $1000 \times 10^{4}$ dyne $/ \mathrm{sec} / \mathrm{cm}^{2}(\mathrm{HE}, \times 80)$. the most difficult problem in the study of rheological characters of matrix in vivo studies is the lack of suitable techniques as well as the diversified composition of matrix. The so-called matrix is astructurally homogeneous, viscous in character and is chemically composed of glycosaminoglycans (GAGs). Even though biological importances have been emphasized, matrices' complicated characters are not only dependent on their biochemical alteration but also on the colloidal chemical properties. It was so difficult to gasp objectively the many characters of matrices in biological phenomena.

Depending on the theoretical basis of histopathology, we made note of the common character of GAGs and set out to study the analysis of their pathognomatic mechanism and its morphogenesis in tissues and organs.

The most common characteristics of GAGs are their polymer structure, consisting of repeated disaccharide units, and their strong negative charges. ${ }^{11}$ When their negative charge is neutralized by cations, GAGs yield a flocculent precipitate. Their molecular structures are condensed, but without complexing to cations, units of GAG molecules repel each other, and the result is a loosened and elongated molecular structure (Fig 4). ${ }^{12}$ Through these characteristics, they display their biological roles; exudation, cellular movement and also in some organs, such as in the glomerulus, where no effective fixing structures such as collagen fibers exist, GAGs may act as the structural elements of reinforcement. This

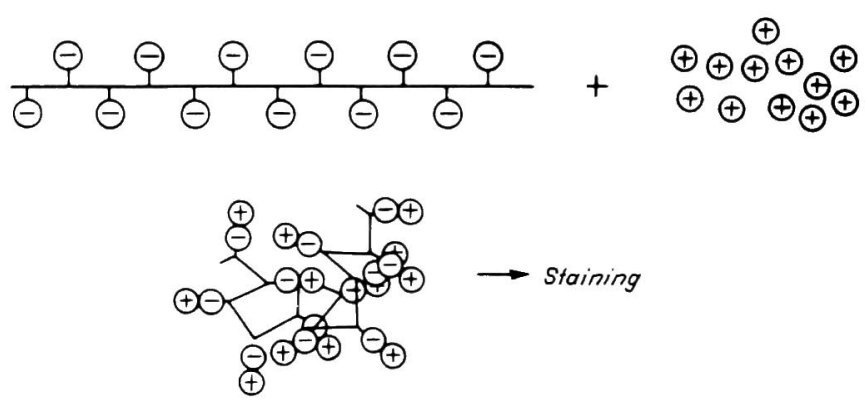

Fig 4 Common principle of staining of GAGs, established by Pearse. This schematic figure shows alteration of the molecular structure of GAGs in relation to the static electron charges.

kind of molecular structural alteration of GAGs will be influenced by their colloid chemical property, the sol-gel diversion of the matrix.

On the other hand, thinking of positively charged materials, mono-charged materials are mainly related to functions, and multi-charged ones to the structures of materials to form the molecular skeleton. However bivalent materials are related to both functions and structures (Fig 5). Generally speaking the functional aspect is more important for survival than structural ones, so when a decrease in the amount of these substances can be induced, they are shifted from the structural to 


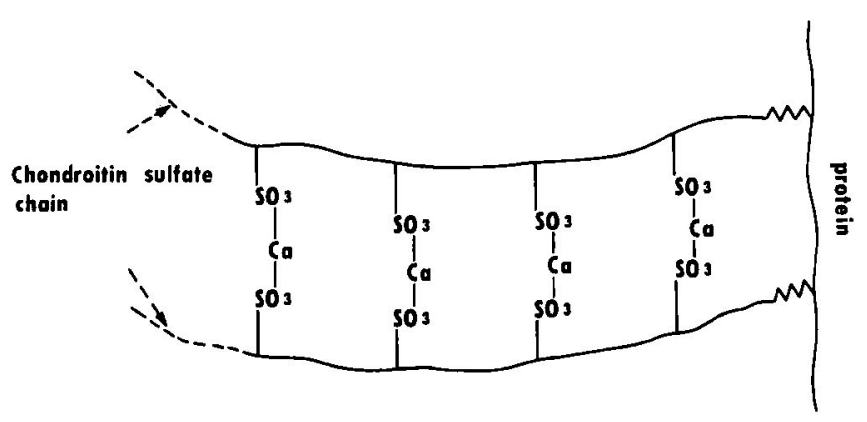

Relation between chondroitin sulfote and $\mathrm{Ca}^{2+}$

Fig 5 This figure shows the relation of molecular structure of GAGs to calcium ion.

the functional phase, resulting in the provocation of some morphological distortions.

Among many bivalent cationic materials, calcium (Ca) is the most common and widely distributed one, which is easily ionized, and is related to both functional and structural aspects of living animals (Fig 5). Considering $\mathrm{Ca}$ in the structural phase, as $\mathrm{Ca}$ complexing to GAGs has strong ionization activities, it is released into the functional phase more easily than the $\mathrm{Ca}$ of the bone. Therefore to maintain homeostasis, a shift of the $\mathrm{Ca}$ ion from structural to functional phase occurs in a fairly short term as a result of which this shift will induce the structural alteration of matrix.

\section{Structural Aspects of Low Calcium Condition}

Animals were bred with low $\mathrm{Ca}$ diet $(0.2 \%$ calcium contained diet) for a maximum of 6 months. With this experimental procedure, duplication of the glomerular basement membrane (GBM) and mesangiolytic changes with balloon-like dilatation of glomerular lumina ${ }^{13}$ were observed which are thought to be the basic and initial morphological alteration of renal lesions (Fig 6). Thus the removal of $\mathrm{Ca}$ ions from the molecular structure of GAGs changed their colloidal chemical property into sol, resulting in a decrease of the viscosity of the mesangial matrix. In renal glomeruli GAGs are the sole factor which preserve the structure and with the loosening of the GAG molecules, mesangiolysis results. In the past various degrees of mesangiolytic changes of the glomeruli have been demonstrated in the autopsied patients who died of acute pancreatitis, in which the blood $\mathrm{Ca}$ level had dropped unusually and rapidly, because of Ca saponification with necrotic fatty tissue. This is the reinforcing and supporting data for the present study. In addition to the mesangiolysis, as mentioned above, laminification of the GBM (Fig 7) predisposed suitable sites for glomerular deposits. ${ }^{14}$ When positively charged materials pass through the negatively charged GBM, tested with cationic ferritin, the result is the conjugation of these oppositely charged molecules. ${ }^{15}$ These mechanisms are common not only to the renal deposits but to the deposition of immune complexes throughout the body.

In these animals in our study who survived for a long period under a low Ca condition, sudden death of the animals was noted in which the liver cell embolisms in

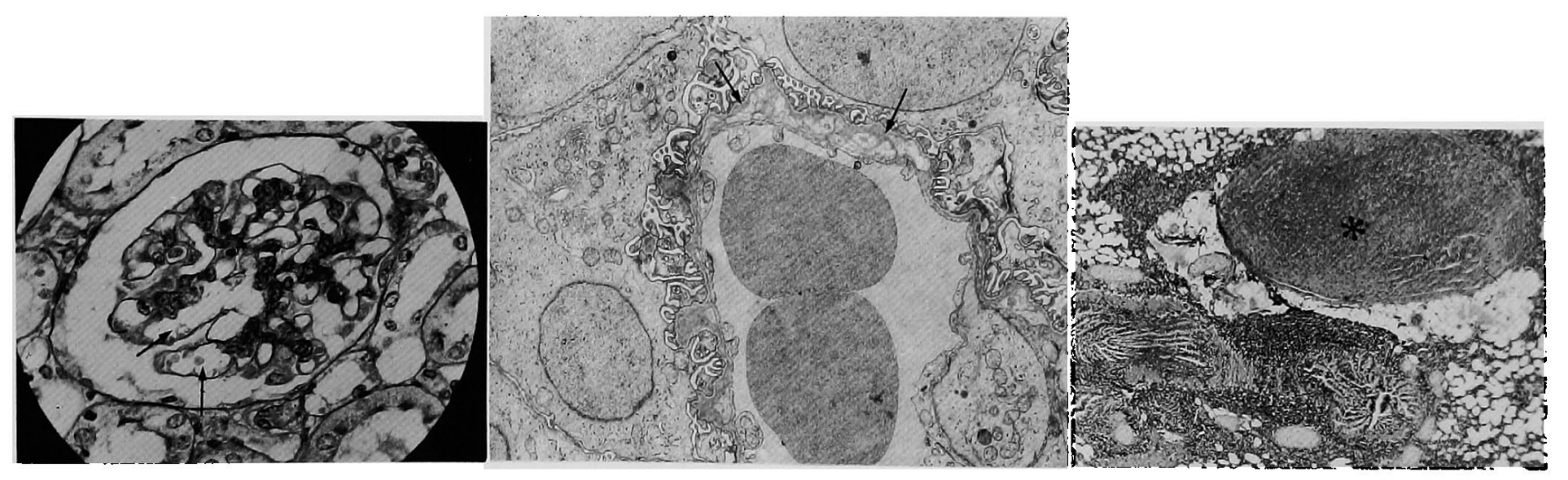

Fig 6 Mesangiolytic changes of glomerular tuft are seen under low calcium diet breeding of the animals, resulted in the balloon-like dilatation of glomerular capillary(arrow). These changes are thought to be the common initially observed morphological manifestation in kidney (HE, ×380).
Fig 7 With EM studies, dilatation of glomerular capillary with duplication and reticulation of GBM arrow) is noted $(\times 8,000)$.
Fig 8 Examination of sudden death cases during breeding by low calcium diet shows cmbolism of liver cell cluster $\left({ }^{*}\right)$ in the pulmonary arteries occasionally (HE, $\times 20$ ). This shows the loosening of the intercellular junction under a low calcium condition. 
the pulmonary arteries (PA) were sometimes noted (Fig 8). This shows that a prolonged low Ca diet, with resulting low $\mathrm{Ca}$ condition, had a serious influence on intercellular junctions. Sialic acid of GAGs is the most general cause of the negative surface charge of the cells and it has been suggested that this has a very important and inevitable influence on cell adhesion. ${ }^{16}$ Formation of $\mathrm{Ca}$ linkages decreases the negative charges of the carboxyl radical and supports the stability of static electricity of the cell surface and, as a consequence cellular adhesion. The cells are coupled by junctions consisting of membrane channels through which molecules of a certain size can flow freely from one cell interior to another. The role of $\mathrm{Ca}$ has been studied mostly under normal conditions. ${ }^{17-19}$ It has been proposed that the permeability of the junctional channels depends on the concentration of free ionized $\mathrm{Ca}$ in the cytoplasm. On the other hand, contact inhibition has already been described by Abercrombie and Heaysman. ${ }^{20-22}$ To sustain this, growth inhibition should inevitably be induced simultaneously by some mechanisms which can be regarded as mitosis inhibition. Contrary to this, the mitotic activity of cells losing contact inhibition expected to continue. In a long-term low Ca state, the extraordinary proliferation of bronchial epithelia with obstructive bronchitis was demonstrated (Fig 9), ${ }^{23}$ but it is not clear whether this morphology depends on the loss of communication through junctional channels, or loss of contact inhibition, or both. In any event, these results stress the importance of the role of the $\mathrm{Ca}$ ion in intercellular conjugation and communication and also in cellular proliferation through loss of contact inhibition.

In addition to the low $\mathrm{Ca}$ diet, rapid decrease of
Ca ions from serum was accelarated by intraperitoneal administration of $\mathrm{Na}_{2}$ EDTA which is usually used as the chelating drug for $\mathrm{Ca}$ and $\mathrm{Mg}$.

With the administration of a single dose of $6 \%$ $\mathrm{Na}_{2}$ EDTA ( $4 \mathrm{ml}$, approximately $0.178 \mathrm{~mol}$.) the animals suffered from tetanic shock from which almost $60 \%$ of the animals died. Postmortem dissection showed that the mesenterial arteries demonstrated severe angiolytic changes (Figs 10, 11). ${ }^{24,25}$ Extremely expanded intermuscular spaces with spindle-shaped smooth muscle cells in the media were stained strongly with alcian blue and colloidal iron staining. Fragmentation of internal elastic lamellae was also seen. When adding immune complexes, fulminant angitis with fibrinoid degeneration was demonstrated (Fig 12). ${ }^{26}$ This fact shows the importance of the predisposition for the provocation of angitis more than exogenic factors. Combined procedures of predisposition and exogenic factors are necessary successfully to provoke the typical angitis. The results obtained suggest one of the possible roles of colloid chemical alteration of GAGs for the formation of fulminant angitis in interpreting the results of the above staining. Angiolytic changes may be displayed by loosening of matrix. After angiolytic changes, with calcium lactate administration, calcification was noted limited to injured areas, initiating from internal elastic lamellae and then spreading up to the vascular media (Fig 13). This means that not only the intercellular matrix but also the elastic lamellae have a strong $\mathrm{Ca}$ affinity. This will be resulted in aneurysmal changes of the vasculatures (Fig 14), ${ }^{27}$ probably by weakness of the vascular wall plus vasomotion.

In a low Ca condition, necklace-like detachment of the endothelial cell layer from the vascular wall (Figs 15,

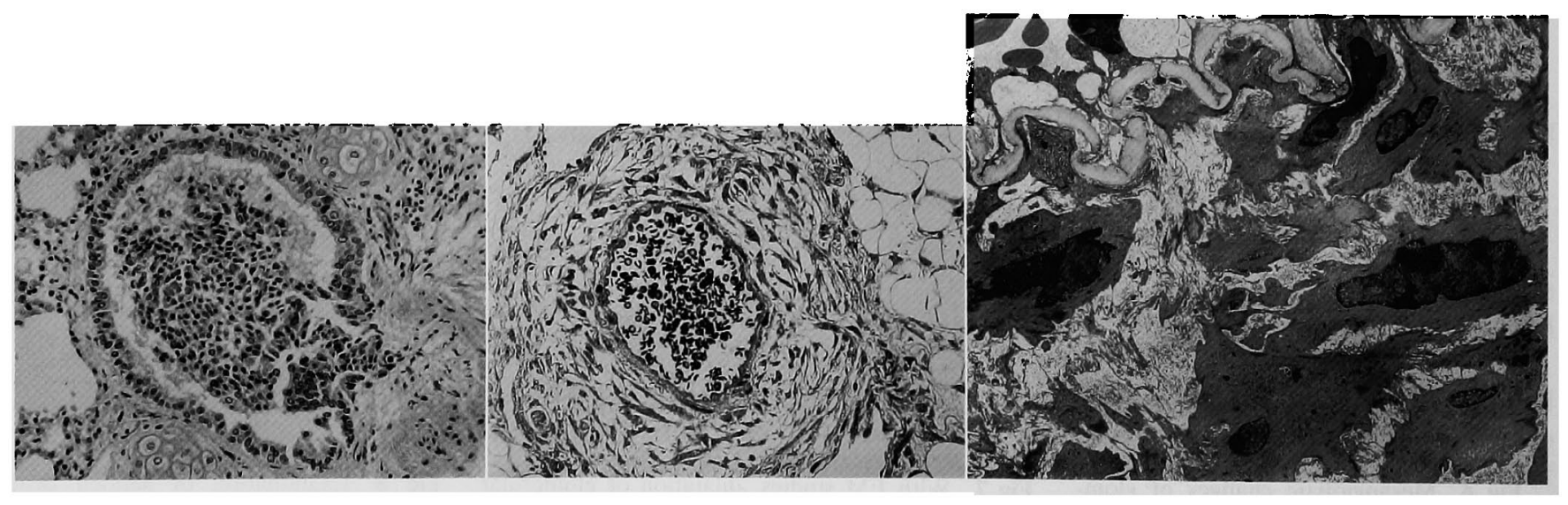

Fig 9 Under a low calcium condition, obstructive proliferation of bronchial epithelia is observed which is thought to be the morphological manifestation of lose of contact inhibition (HE, $\times 400$ ).
Fig 10 With administration of $4 \mathrm{ml}$ of $6 \% \mathrm{Na}_{2}$ EDTA intraperitoneally once, severe angiolytic changes of mesenterial arteries are demonstrated $7-10$ days after $(\mathrm{HE}, \times 80)$.
Fig 11 EM study reveals the expansion of intermuscular spaces with spindle shaped smooth muscle cells. Vacuolar formation and lift up phenomenon of endothelial cells with fragmentated and worm eatened-like internal elastic lamellae are also noted $(\times 2,300)$. 


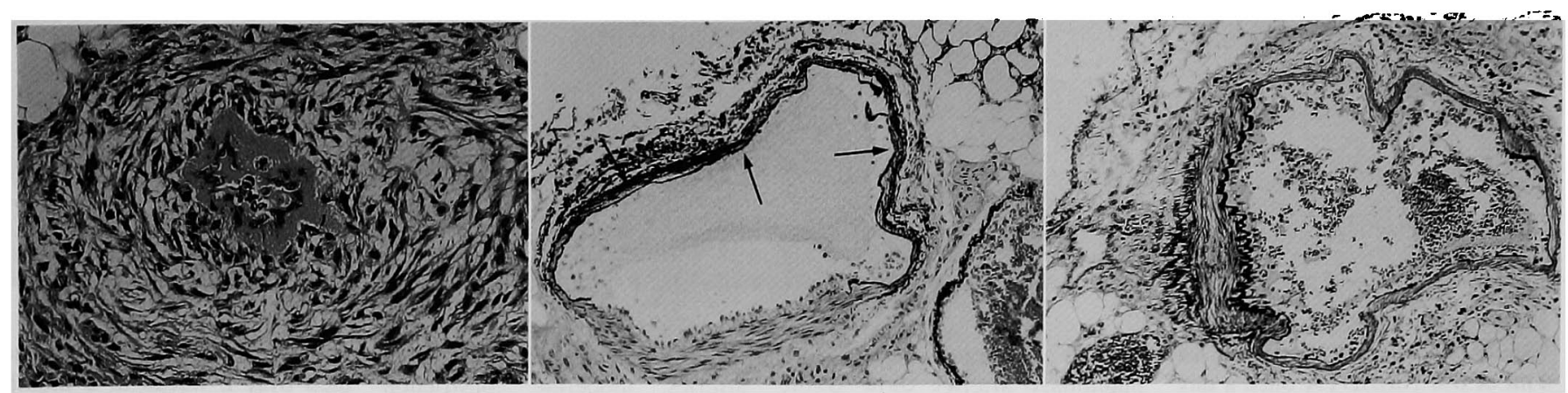

Fig 12 Adding to the above procedures, immune complex is injected and the fulminant angitis with fibrinoid like degenerations is obtained $(\mathrm{HE}, \times 80)$.
Fig 13 Adding to the above procedures, calcium lactate solution is administered and calcification is seen limitedly in the injured areas(arrow) but not in less damaged areas. Kossa stain $(\times 180)$.
Fig 14 Remaining the less damaged areas as an axis, aneurysmal dilatations are noted. Elastica-Van Gieson stain $(\times 180)$.

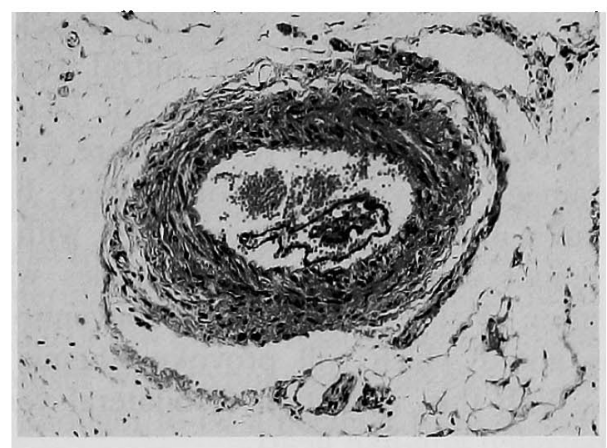

Fig 15 Fairly long survived cases show necklace like detachment of endothelial cell layer without disruption from the vascular wall. There is noted loosening of the vascular wall also. This means that calcium ion does not give the influence to the interendothelial junction but to those of endothelial cell adherence to the vascular wall (HE, $\times 150)$.

$16)^{28}$ was noted, accompanied by degenerative changes of internal elastic lamellae and also positive staining of the vascular wall by colloidal iron staining. It is recognized that a low $\mathrm{Ca}$ condition does not have any influence on interendothelial junction.

\section{Functional Aspects of Low Calcium Condition}

Starting our research from a different experimental viewpoint, we decreased the $\mathrm{Na}_{2}$ EDTA dose in order to prevent typical tetanic shock, and expected this dose to induce mild constriction of the muscle cells.

Administration of a small amount of $\mathrm{Na}_{2} \mathrm{EDTA}$ intraperitoneally provoked animal death after 10 days to 2 weeks over a 2 month period. In the initial phase of 10 days to 2 weeks, rupture of the right ventricular wall along the septum occurred at an anterior angle with

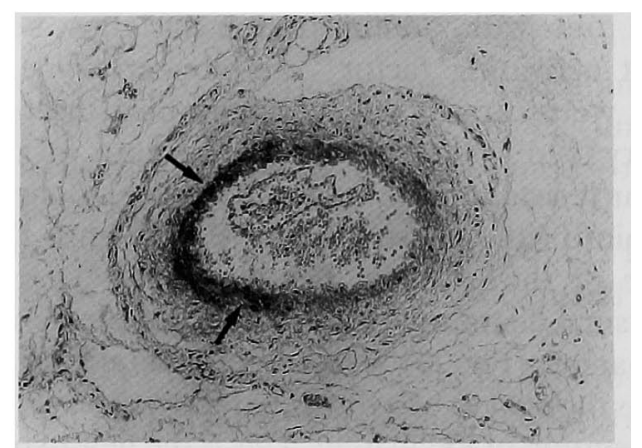

Fig 16 Detached vascular walls are stained strongly positive with colloid iron staining(arrow). This means not only internal elastic lamcllae but also intermuscular matrix are related to the adherence of endothelial cells to the wall $(\times 150)$.

moderate right ventricular dilatation and a remarkably thin ventricular wall (Fig 17).

Characteristic of these cases was elastosis in the main pulmonary arteries (PA) accompanied by a severe degree of constriction (Figs 18, 19). On the other hand, severe dilatation of the right ventricular lumina with paper-like thinness of the wall (Fig 20) was demonstrated in the delayed cases of more than 2 weeks to 2 months.

In these cases, the main PA showed rather slight constriction with mild elastosis (Fig 21). Conversely, the peripheral PA showed extraordinary constriction with marked vacuolar formation in the media where red cells could hardly pass through (Fig 22).

These results confirmed that actual constriction at various sites of the PA, resulting in extraordinary pulmonary hypertension (PH), basically plays an essential role in the provocation of these changes. Also considering the effects on the heart, depending on the constriction site of the PA, alternatives could include rupture or not. 


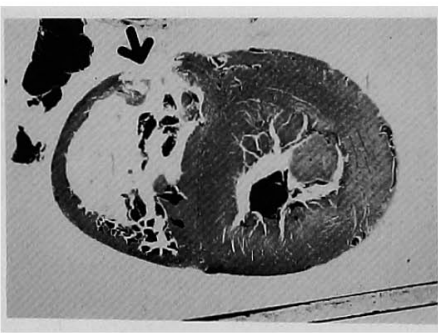

Fig 17 With administration of $1 \mathrm{ml}$ of $6 \% \mathrm{Na}_{2}$ EDTA once a day for 2 months, the animals died within 2 weeks show the moderate dilatation of right ventricle with rupture at an anterior angle(arrow) (HE, $\times 6$ ).

Experimental studies on the provocation of $\mathrm{PH}$ were carried out with administration of glass beads, ${ }^{29-33}$ platelet activating factor ${ }^{34}$ or monocrotaline. ${ }^{35}$ These studies were carried out by diminishing the pulmonary capillary bed which led to an increase in peripheral vascular resistance. No studies have been done from the viewpoint of the constriction of PA which is thought to be one of the essential factors of PH. In our study, aneurysmal dilatation (Fig 23) and also plexiform lesionlike changes (Fig 24) were experimentally encountered which is thought to be a most characteristic morphological manifestation of PH in lung. This is the new experimental model for the provocation of $\mathrm{PH}$. In those animals with long survived ones, similar morphology was noted to idiopathic dilated cardiomyopathy (IDC) (Fig 25) probably induced by pulmonary distress ${ }^{36}$ which has never been provoked by experimental procedures. A number of authors have described IDC patients with $\mathrm{PH}^{37-44}$ According to Iijima, ${ }^{45}$ the present finding related to pulmonary vasculature indicates that patients with IDC may have venous $\mathrm{PH}$ in the late stage and he noted widespread myocardial fibrosis in all patients with IDC

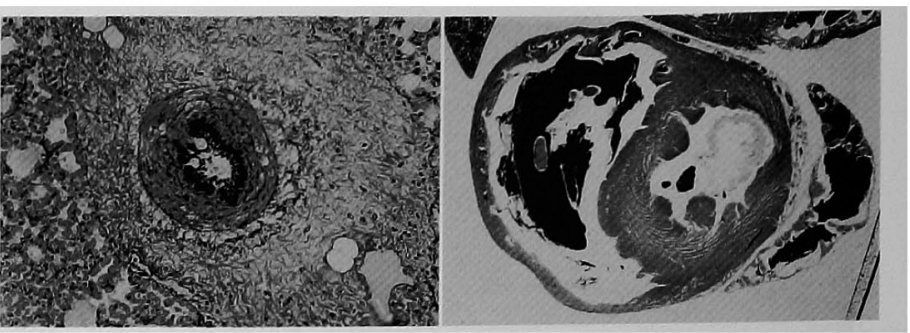

Fig 19 In peripheral pulmonary arteries, moderate constriction with vacuolar formation in the media is noted. Elastica-Van Gieson stain $(\times 130)$.
Fig 20 On contrary, in animals died over 2 weeks to 2 months, extraordinary dilatation of the right ventricle with paper-like thinness of the wall is demonstrated (HE, $\times 6)$. with marked dilatation of the left ventricles which is frequently demonstrated in papillary muscles..$^{40,42-44}$ Romeo $(1989)^{46}$ suggested that mitral regurgitation may be one of the important prognostic factors in this disease. In our experimental results, there was no myocardial damage and valvular incompetency, but on the other hand a marked constriction of PA with collapse, severe venous congestion and sometimes widespread consolidation of alveolar areas with conventional infection was noted which will provoke ishemia in myocardium. With this, dilatation of bilateral ventricles is double barreled gun-like heart.

\section{Unclarified Morphological Variations by Durative and Fairly Mild Low Calcium Condition}

These experiments were done using practically insoluble $\mathrm{Ca}$ chelating agents, therefore very mild effects of low Ca were given for longer period. Long-lasting effects showed a range of morphological manifestations, the pathogenesis of which are difficult to explain. With long term observation, remarkable hyperplasia of the

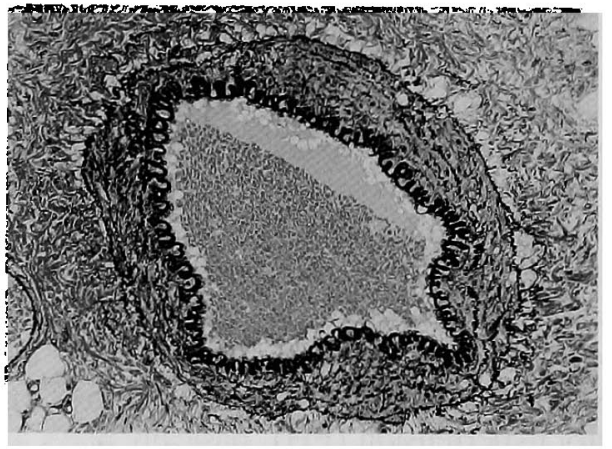

Fig 21 In the main pulmonary arteries, moderate constriction with scare elastosis is seen. ElasticaVan Gieson stain $(\times 200)$.

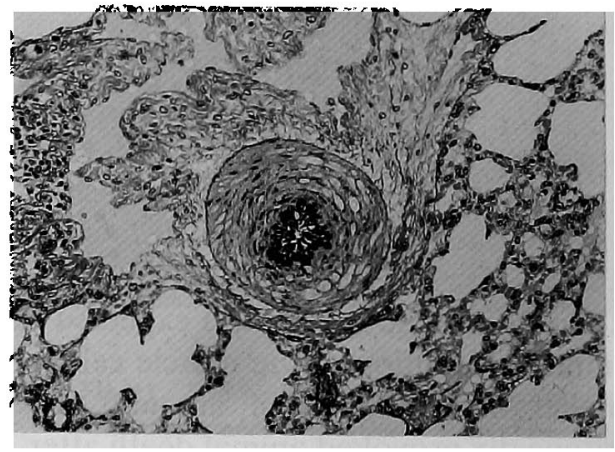

Fig 22 In peripheral arteries, severe constriction which could hardly pass a red cell is seen. ElasticaVan Gieson stain $(\times 200)$. 


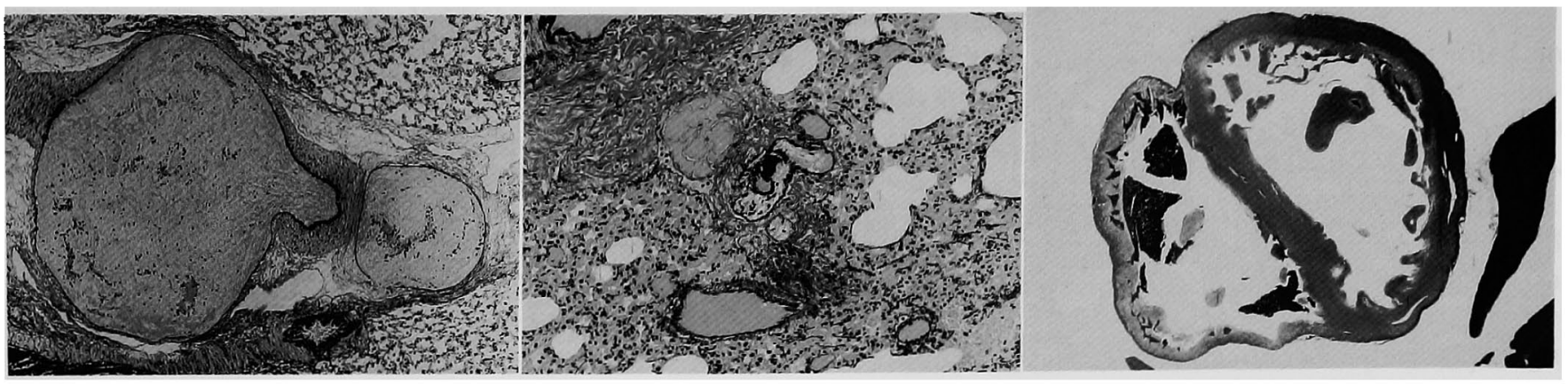

Fig 23 Formation of balloon-like dilatation of the pulmonary arteries is observed. Elastica-Van Gieson stain $(\times 60)$.
Fig 24 Plexiform-like lesion which noted triple divided cavities is noted. Elastica-Van Gieson stain $(\times 150)$
Fig 25 Bilateral dilatation of ventricles, accompanied by pulmonary distress. This morphology is similar to those of idiopathic dilated cardiomyopathy (HE, $\times 6)$. juxtaglomerular cells was seen (Fig 26).$^{47}$ It is the most characteristic morphological manifestation of Bartter's syndrome which is a rarely seen genetic disorder. However, it is of specific interest with respect to the hormonal excretion of the kidney, including renin, angiotensin and aldosterone as well as biological alteration of the various electrolytes. This is thought to be based on juxtaglomerular hyperplasia, very high plasma renin with normal or low blood pressure, and secondary hyperaldosteronism with hypokalemic alkalosis. The disease has been thought to be a peculiar form of salt wastage. The pathognomatic mechanism of this disease is that the renin-angiotensin-aldosterone system is activated by chronic volume constriction, but the salt-wasting state results in a continuous delivery of sodium salts to the distal nephron, producing hypokalemic alkalosis.

Hitherto total $\mathrm{Ca}$ level in the serum has not been considered to be essential. Trygstad $e t$ al $^{48}$ reported that two out of three patients examined featured a temporary increase in the Ca level in serum whereas the other case demonstrated $\mathrm{Ca}$ discharge from the bone resulting in fracture. Arant $e t$ al ${ }^{49}$ presented similar cases. Taugner et $a l^{50}$ announced that three cases out of five demonstated hypercalciuria, in which the urinary content of $\mathrm{Ca}$ was almost three times more than normal. These cases also demonstrated nephrocalcinosis. Nonetheless, Ca balance is presently thought not essential for the provocation of Bartter's syndrome. We hope that the above consideration will help to clarify this rarely seen but very interesting syndrome. Electrolyte imbalance in these conditions in relation to hormonal aspects should be studied in this disease. Furthermore, frequent and simultaneous observation of peripolar cells (Fig 27) ${ }^{51}$ which are thought to play a role in sodium control of urine is noted. The exact reasons of this hyperplasia have not been clarified.

\section{Interim Summary for the Future Studies}

In this paper, we have reviewed the morpho-functional alterations under various modes of low calcium conditions and at present the exact pathogenesis of these manifestations has not yet been clarified.

Considering the above results obtained under the low Ca conditions renal lesions will be discussed as an example. ${ }^{52,53}$

Pathogenesis of the diseases should be considered as an integrated phenomenon combining endogenous and exogenous factors. However, with a few exceptions, it is extremely difficult to explain the relationships between a disease and the predisposition that play a key role in the genetic background.

We have carried out our research in order to clarify the relationship between a disease and the predisposition, by trying to define the relevant phenotype in the genetic background of the disease, thereby elucidating a formative mechanism for provocation of the disease. Through this research, we intended to reveal a positive role of the extracellular matrix, including the basement membrane (which in the past has been regarded merely as a supportive tissue), within the framework of a variety of pathological changes. It has been established that the administration of anti-renal, anti-pulmonary or antiaortic serum is exclusively accompanied by the development of glomerulonephritis with no manifestation of organ specific changes such as pulmonary or aortic lesions. This certainly raises questions about the validity of the conventional concept which stresses the role of allergy in provocation of nephritis and denies explanation of the disease on the basis of an organ allergy theory. ${ }^{54}$

The concept of nephritis as used in diverse, and it is frequently difficult to make a differential diagnosis pathologically during the initial stages of lupus nephritis. IgA nephritis, purpura nephritis, renal lesion in Fabry 


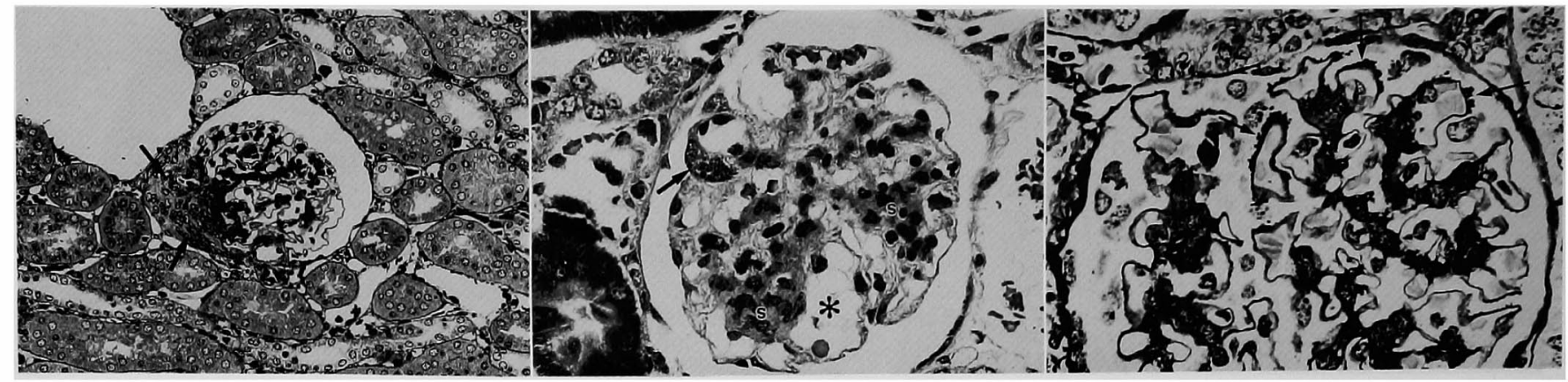

Fig 26 Extraordinary hyperplasia of juxtaglomerular cells(arrow) which is compatible to $1 / 3$ of the glomeruli. PAM stain. This figure is identical to the basic morphology of Bartter's syndrome $(\times 240)$.
Fig 27 Frequent detection of peripolar cells(arrow) is seen. In this figure, also mesangiolytic changes(*) and segmental proliferation of mesangial cells(s) are demonstrated. MSB trichrome stain $(\times 480)$.
Fig 28 Kidney, 5-6 months after Quinn II administration. Cystic dilatation of the glomerular capillary lumina due to masangiolysis and segmental proliferation of the mesangial cells are noted. Severe and widespread spike-like projection with an irregular tickening of GBM(arrow) are also demonstrated. PAM, stain $(\times 800)$. disease and diabetic nephropathy, as well as in the more narrowly defined glomerulonephritis. Therefore, from a morphological point of view, we must take a broad perspective, and not confine ourselves to the narrowly defined idea of inflammation set forth by Jones ${ }^{55}$ in his "Inflammation and Repair of the Glomerules". Furthermore, it may be for these reasons that the ratio of immune complex (IC) nephritis to all renal diseases should be reconsidered.

Shibata $e a{ }^{56,57}$ stress the importance of non-immunological complex for the provocation of nephritis. According to those authors, when repetitive deposits of this non-immunological complex in BM exceed a certain level, repair processes are invoked, and this in turn leads to the pathological manifestations of chronic renal disorder.

More recently, the importance of basement membrane $(\mathrm{BM})$ in biological phenomena has been discussed in many scientific fields. Here again we should notice that the etiology of these kinds of diseases has been dealt with traditionally on the basis of an immunological standpoint entirely independent of the concept of the extracellular matrix, which cannot be ignored. However, nowadays more than a few researchers have found that an IC-induced pathological change may cause a renal disorder of reversible and transient nature, but that in case of a chronic and progressive type of renal lesion, a different process based on the alteration of the extracellular matrix including the BM, should be considered.

According to the noteworthy works of Shibata et al,${ }^{56,57}$ new approach in the explanation of the limited pathological mechanism should be offered. However his works are hardly adequate to explain the formative mechanism of various deposits featuring extensive and diversified properties in the extracellular matrix, including GBM.

With this in mind, we tried to find a more common mechanism where extensive and diversified processes in the depositing of miscellaneous substances take place in the glomerulus, from an endogenous viewpoint. For that purpose, alteration of the molecular structure of the extracellular matrix has been emphasized, especially with regard to its electron charges. Therefore, the structure which was thought to be linked to those phenomena in the provocation of morphological changes, was experimentally altered. We chose GAGs purely as an example for the experimental hypothesis. For example, the BM is composed not only of GAGs but also of glycoprotein, namely laminin and fibronectin, and type IV collagen. $\mathrm{Ca}$ ion is involved in their functions and structures as well as in their inter-binding and binding with cells.

On the other hand, $99 \%$ of $\mathrm{Ca}$ is found in the bones, and the remainder exists in the serum as well as in the intra- or extracellular components. The Ca levels in structures other than bone and those related to cellular functions, generally range from $10^{-3}-10^{-7} \mathrm{~mol}$. With regard to the $\mathrm{Ca}$ level in living animals, it is more appropriate to assume that there are 2 different types of $\mathrm{Ca}$, one related to functions and one involved in structures.

In an attempt to clarify the role of $\mathrm{Ca}$ ion in biological phenomena, we carried out an experiment of which the design was formulated by taking into account varied characteristics such as long-term feeding with a low $\mathrm{Ca}$ diet, calcitonin, $\mathrm{Na}_{2}$ EDTA and Quinn II administration: Our results in relation to matrix are summarized below.

A low Ca diet over an extended period of time, with a relatively mild decrease in the $\mathrm{Ca}$ ion content, was accompanied by mesangiolysis of the glomeruli and reticulation of their BM. 
A moderate state of reduced $\mathrm{Ca}$ due to calcitonin administration was accompanied by the development of a pathological changes resembling IgA nephrophathy.

An abrupt and transient $\mathrm{Ca}$ ion decline of a severe degree ensuing the administration of $\mathrm{Na}_{2} \mathrm{EDTA}$ was associated with severe systemic symptoms such as tetanic shock and angiolysis at the site of administration.

The common objective of these trials was to see what kind of pathological changes would be introduced via the extracellular matrix when it was heavily loaded with negative charges as a result of the elimination of $\mathrm{Ca}$ ions from the molecular structure of GAGs.

In case of Quinn II administration, because the agents dissolve very slowly in water, it is assumed that they dissolve in the perfusing peritoneal fluid gradually and slowly over a long-term period, and that when the dissolved chelating agents are discharged from the kidney, they exerts a durative effect on the small amount of $\mathrm{Ca}$ ions that is regarded important in retaining the structure of the glomerulus, and at the same time it affects a change in the molecular structure of GAGs which are an important component of the extracellular matrix.

Mesangiolysis or reticulation of GBM (Figs 6, 7) occurred, which is a common morphological manifestation in the initial stage of renal lesions. Such changes, if advanced, resemble the splitting or reticulation which has been documented in a series of renal lesion, including IgA or membranous nephropathy, purpura nephritis and Alport's syndrome. The meaning of such provoked changes in the kidney poses a problem to be re-evaluated through further work designed to find a common denominator in these diseases. The most interesting finding is that the changes resemble a lesion caused by autosomal dominant inheritance in hereditary nephropathy (Alport's syndrome). All in all, this, along with the elucidation of the phenotypic mechanism governing pathogenesis in these diseases, is certainly a crucial question for future research.

The formation of a spike along BM which will be followed by an irregular protrusion on the epithelial side in the form of a meshwork which houses a fine granular structure inside (Fig 28). These extreme form of the duplication and reticulation in the GBM are demonstrated by Alport's syndrome, $\operatorname{IgA}$ nephropathy and purpura nephritis. On this assumption, we named this change in the GBM "reticulo-protrusion". A deposit can also be located on the epithelial side of the GBM, and the observed foot process fusion and the proteinaceous insudation into the epithelial cells and striking hyaline droplet degeneration in the proximal tubules, suggested the existence of severe proteinuria, which apparently functionally impairs glomerular filtration.

At time passes, the lesion appears as something like an irregular thickening of GBM rather than a spike, although a washout phenomenon of a deposit can some- times be seen.

In brief, this change may resemble that noted in membranous nephropathy. However, the term membranous nephropathy, in the strict sense, can hardly be applicable in this case, since the pathogenesis is time-phased and characterized by initial reticulation, protrusion toward the epithelial side and a deposit located in that area. Nonetheless, the possibility of progression to the status of membranous nephropathy can hardly be ruled out when one considers the exceptionally high incidence of deposits of C3 (Fig 30) and IgG (Fig 29).

Electron microscopic studies detected deposits in the paramesangial areas (Fig 35). Morphologically, it is quite similar to $\operatorname{IgA}$ nephropathy but immunohistochemical technique proves that $\mathrm{C} 3$ and $\mathrm{IgG}$ are strongly stained and that IgA (Fig 32) and IgM (Fig 31) are not stained.

Experimental renal lesions associated with Quinn II which begin in the form of mesangiolysis, show various morphological manifestations depending on the time element, and no comparable pathological changes are documented to have naturally occurred in the human kidney. In some respects, it is similar to Alport's syndrome (Fig 33), and in others it reminds one of an entity progressive to membranous nephropathy (Fig 34). However, in yet other respects it is similar to IgA nephropathy (Fig 35). Given that lesions result from changes occurring through alteration of the molecular structure of GAGs, and that they are caused by sustained mild reduced levels of calcium in the blood, is a highly interesting point from which to consider the common properties of morphological changes in the initial renal lesion, some of which are classified as minimal changes, which have, at times defined morphological differentiation. The question is whether a renal patho-morphological change progresses entirely as an independent change or is caused by a difference in the tissue phenotype brought about by an event occurring during the course of the process. The problem to be discussed is the conformity of highly diversified findings with systematic and conventional classification of the renal diseases. This argument gains further authenticity when we consider that all these findings were results obtained under conditions completely precluding the involvement of IC which is the basis of allergy and immunological theories which dominate the hypothesis of formative mechanisms accountable for the development of nephritis.

Also notably conflicting with the conventional results, are the finding ensuing probing with the immunohistochemical technique. Namely, the paramesangial deposit typifies the precipitation formula characteristics of $\operatorname{IgA}$ nephropathy morphologically, while contradictorily the deposit is found to be composed of $\mathrm{C} 3$ or IgG in the absence of IgA. This finding brings into question the fundamental attitude toward the con- 


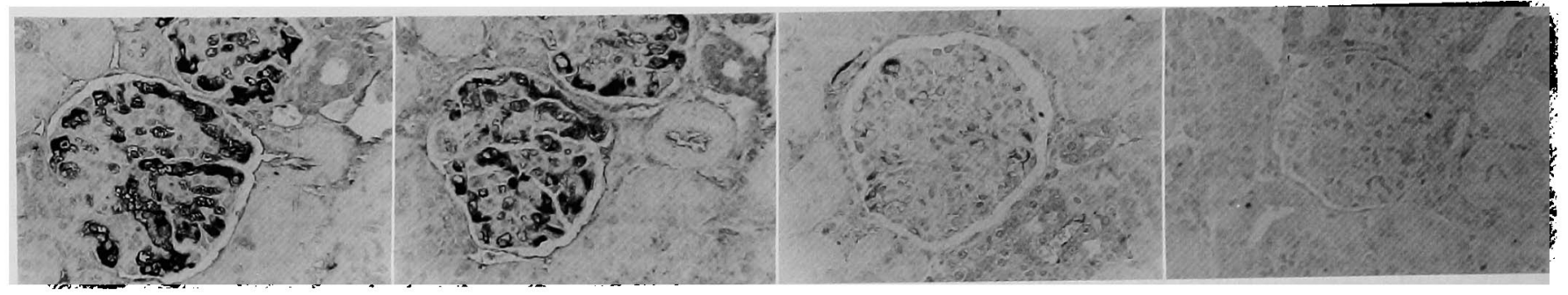

Fig 29 IgG deposits are clearly visible in almost all mesangial areas of glomeruli and slightly presented along the capillary BM. PAP $(\times 400)$.
Fig $30 \mathrm{C} 3$ shows the same figure as above. PAP $(\times 400)$.
Fig 31 In the same slide, IgM is not demonstrated. PAP $(\times 400)$.
Fig 32 IgA is also not demonstrated. PAP $(\times 400)$.

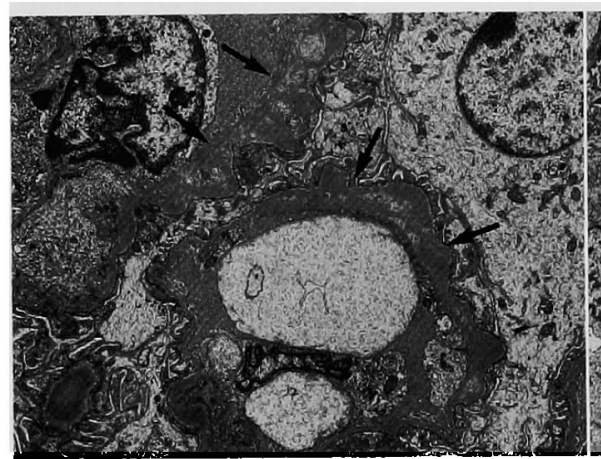

Fig 33 The GBM forms an irregular reticuloprotrusion with thickening and interstitial spaces of the reticulated GBM(arrow) showing a fine granular structure. This figure is similar to Alport's syndrome $(\times 6,200)$.

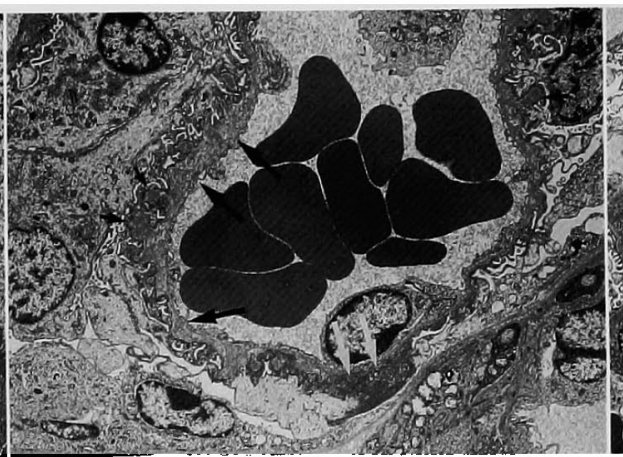

Fig 34 Polypoid protrusion of GBM (small black arrow) with deposits toward the subendothelial side and diffuse duplication (large black arrow). White arrows show proteinous insudation into epithelial cell. This figure is similar to membranous nephropathy $(\times 3,300)$.

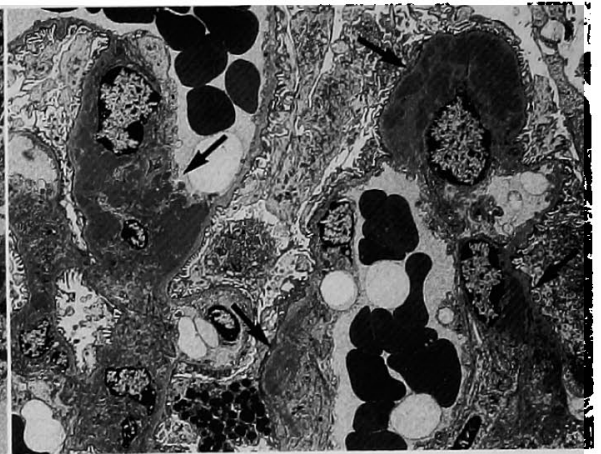

Fig 35 Dense deposits are noted in the para-mesangial area of all the glomeruli, 6 months later. This morphology is similar to IgA nephropathy $(\times 1,800)$. ventional classification of renal diseases which attaches a reasonable degree of importance to deposits. The crux of the matter may be whether it is a deposit inseparable from a tissue phenotype or an accidental phenomenon based on a mere potential difference. The intensity and duration of the state of low Ca may be a case in point, if one considers that morphological changes resembling IgA nephropathy is noted in a slightly more intensive state of low $\mathrm{Ca}$. It follows then that this will be a starting point for new research designed to define the meaning of the electrical charge as a phenomenon occurring between hyperfiltration and tissue pre-disposition.

The observed diversity of renal lesions not only indicates the common denominator of the genetic background responsible for the development of pathological changes in the kidney, but also suggests that the involvement of secondary factors such as hyperfiltration and electrical charge in the process of diversification of the changes is great.

These experiments were carried out in order to eluci- date the common denominator involved in the diversity of morphological phenotypes and the status of the disease, through elucidation of the phenotypes of the genetic predisposition that more or less influence the development of renal lesions. Various experimental techniques imply a difference in the degree of involvement of the predisposition of a particular morphological phenotype. They further remind us that the intensity of the deposit and the diversity of the materials are not necessarily due to the product of an immunological process, but rather are problems requiring consideration from the viewpoint of potential difference in the charges found between the deposit and the host. The results obtained from these experiments have led to a new approach to the genetic background and its essential properties which have formerly been ignored. We believed that the findings will also give important information for reviewing the directions followed in the past, which gave more emphasis to exognous factors as an explanation for the mechanism of the development of the pathological changes 


\section{than endogenous ones.}

As mentioned above, we think that quantitative and qualitative alterations of GAGs sometimes form the background of the various diseases, and of their diversified phenotypes in the morphological manifestations. The various results obtained through our experimental works were reviewed in this paper. It is clear that $\mathrm{Ca}$ ion is merely one example to provoke the molecular structural alteration of GAGs but the genetical properties, such as ion trapping capacity or the degree of negative charge they have, are also essential. We use low $\mathrm{Ca}$ condition in our studies based on the premise that the $\mathrm{Ca}$ ion is used as a tool to induce morphological manifestations; therefore analytic studies of the hereditary diversity of GAGs should be done in the future.

A lot of facts obtained remain unsolved, which should be studied more both morphologically and functionally. However, here we could stress examination of the important and inevitable role of the matrix which has been rather ignored. We would like to step out into new ways to analyze the pathogenesis of these diseases and to stress the important role of $\mathrm{Ca}$ in biology.

\section{References}

1. Boyden S: The chemotactic effect of mixtures of antibody and antigen on polymorphonuclear leucocytes. J Exp Med 1962, 115: 453-466

2. Keller HU, Sorkin E: Studies on chemotaxis. 13. Differences in the chemotactic responses of neutrophil and eosinophil polymorphonuclear leucocytes. Int Arch Allergy Appl Immunol 1969 , 35: $279-287$

3. Stecher VJ, Sorkin E: Studies on chemotaxis. XII. Generation of chemotactic activity for polymorphonuclear leucocytes in sera with complement dificiencies. Immunology 1969, 16: 231-239

4. Young JS: The invasive growth of malignant tumours: a experimental interpretation based on clastic-jelly models. J Pathol Bacteriol 1959, 77: 321-339

5. Ward PA, Newman LJ: A neutrophil chemotactic factor from human C'5. J Immunol 1969, 102: 93-99

6. Takeuchi H: Studies on the behaviour of peritoneal macrophages of guinea pigs against various foreign materials using the "agar bullet" method. Acta Pathol Jpn 1973, 23: 35-42

7. Yamaguchi H, Nakajima S, Takeuchi H, Torikata C, Kageyama $\mathrm{K}$ : Studies on the behavior of peritoneal cells against soluble immune complexes using the "agar-bullet" method. Exp Mol Pathol 1973, 19: 320-327

8. Yamaguchi H, Takeuchi H, Torikata C, Ashizawa M, Shiobara $\mathrm{H}$ : Rheological studies of matrix-special reference on the mobility of leucocytes in relation to the rheological characters of experimental matrix. Exp Pathol 1977, 14: 259-272

9. Fricdman $\mathbf{H H}$, Whitncy JE, Szczesniak AS: The textrometer-a new instrument for objective texture measurement. J Food Sci 1963, 28: $390-396$

10. Szczesniak AS: Classification of textural characteristic. J Food Sci 1963, 28: 385-389

11. Meyer K, Davidson E, Linker A, Hoffman P: The acid mucopolysaccharides of connective tissue. Biochim Biophys Acta 1956, 21: 506-518

12. Pearse AGE, ed: Histochemistry: Theoretical and Applied. 2nd
Ed, London, Churchill, 1960

13. Yamaguchi H, Takeuchi H, Torikata C, Sakaguchi H: Studies on the formative mechanism of mesangiolytic changes of renal glomerulus induced by a low calcium diet breeding. Exp Pathol 1978, 15: $153-160$

14. Yamaguchi H, Torikata C. Takeuchi H, Sakaguchi H: Mechanisms of immune deposition in relation to a loosened molccular structure of acid mucopolysaccharides (aMP) caused by a low calcium diet-with special regard to their negative charges. Exp Pathol 1978, 15: 222-230

15. Yamaguchi $\mathbf{H}$, Liou HT, Morisada $M$, Sakaguchi $H$ : Conjugation of cationized ferritin in renal glomeruli after administration of $\mathrm{Na}_{2}$ EDTA. Exp Pathol 1988, 34: 85-88

16. Weiss L: Sialic acid as a structural component of some mammalian tissue ccll surfaces. Nature 1961, 191: 1108-1109

17. Loewenstein WR, Nakas M, Socolar SJ: Junctional membrane uncoupling. Permeability transformations at a cell membrane junction. J Gen Physiol 1967, 50: 1865-1891

18. Nakas M. Higashino S, Loewenstcin WR: Uncoupling of an epithclial cell membrane junction by calcium-iron removal. Science 1966, 151: 89-91

19. Penn RD, Loewenstein WR: Uncoupling of a nerve cell membrane junction by calcium-iron removal. Science 1966, 151: $88-89$

20. Abcrcrombie M, Heaysman JEM: Observations of the social behaviour of cells in tissue cuture. II. Monolayering of fibroblasts. Exp Cell Res 1954, 6: 293-306

21. Abercrombie M, Heaysman JEM. Karthauser HM: Social behaviour of cells in tissue culture. Ill. Mutual influence of sarcoma cells and fibroblasts. Exp Cell Res 1957, 13: 276-291

22. Abercrombic $\mathrm{M}$, Ambrose $\mathrm{EJ}$ : Interference microscope studies of cell contacts in tissue culture. Exp Ccll Res 1958, 15: 332-345

23. Yamaguchi $\mathrm{H}$, Usui $\mathbf{H}$, Torikata $\mathrm{C}$. Tajima $\mathrm{T}$ : Studies on the proliferation of bronchiolar epithelia following administration of high dose of NaEDTA. Exp Pathol 1980, 18: 477-486

24. Yamaguchi $H$, Usui $H$, Tajima $T$ : Multiple angitis of mesenterial arteries in guinea pigs induced by intraperitoneal administration high doses of $\mathrm{Na}_{2}$ EDTA. I. Light microscopic study. Exp Pathol 1981, 20: 26-30

25. Yamaguchi $H$, Usui $H$, Tajima T, Sakaguchi $H$ : Multiplc angitis of mesenterial arteries in guinea pigs induced by intraperitoneal administration of high doses of $\mathrm{Na}_{2}$ EDTA. II. Electron microscopic study. Exp Pathol 1981, 20: 31-40

26. Yamaguchi $\mathbf{H}$, Morisada $\mathbf{M}$ : Experimental study on fulminant angitis with fibrinoid-like degencration. Exp Pathol 1985, 28: $177-180$

27. Yamaguchi $H$, Tajima $T$, Morisada $M$ : Experimentally induced aneurysms of mesenterial arteries. Studies on the histogenesis of acquired aneurysm. Exp Pathol 1983, 23: 197-200

28. Yamaguchi H, Morisada M, Kaku H, Onodera T, Kurokawa R: Detachment of endothelial cell layer from arterial wall under a low calcium condition. Exp Toxic Pathol (in press)

29. Shelub I, van Grondelle A, McCullough R, Hofmeister S, Reeves JT: A model of embolic chronic pulmonary hypertension in the dog. J Appl Physiol 1984, 56: 810-815

30. Zwissler B. Forst H, Ishii K, Messmer K: A new cxperimental model of ARDS and pulmonary hypertension in the dog. Res Exp Med 1989, 189: 427-438

31. Delcroix M, Melot C, Vachiery JL, Lejeune P, Leeman M, Vanderhoeft P. Naeije R: Effects of embolus size on hemodynamics and gas exchange in canine cmbolic pulmonary hypertension. J Appl Physiol 1990, 69: 2254-2261

32. Dervin G, Calvin JE Jr: Role of prostaglandin $\mathrm{El}$ in reducing pulmonary vascular resistance in an experimental model of acute lung injury. Crit Carc Mcd 1990, 18: 1129-1133

33. Prielipp RC, McLean R, Rosenthal MH, Pcarl RG: Hemodynamic 
profiles of prostaglandin EI, isoproterenol, prostacyclin and nifedipine in experimental porcine pulmonary hypertension. Crit Care Med 1991, 19: 60-67

34. Ohar JA, Pyle JA, Waller KS, Hyers TM, Webster RO, Lagunoff D: A rabbit model of pulmonary hypertension induced by the synthetic platclet-activating factor acetylglyceryl ehter phosphorylcholine. Am Rev Respir Dis 1990, 141: 104-110

35. Kay JM, Suyama KL, Keane PM: Failure to show decrease in small pulmonary blood vessels in rats with experimental pulmonary hypertension. Thorax 1982, 37: 927-930

36. Yamaguchi H, Kaku H, Onodera T, Kurokawa R, Morisada M: Experimental idiopathic dilated cardiomyopathy under low calcium condition. Exp Toxicol Pathol (in press)

37. Massumi RA, Rios JC, Gooch AS, Nutter D, De Vita VT, Datlow DW: Primary myocardial disease: report of fifty cases and review of the subject. Circulation 1965, 31: 19-41

38. Dye CL, Genovese PD, Daly WJ, Bchnke RH: Primary myocardial disease. II. Hemodynamic alterations. Ann Intern Med 1963, 58: 442-453

39. Sackner MA, Lewis DH, Robinson MJ, Bellet S: Idiopathic myocardial hypertrophy. Am J Cardiol 1961, 7: 714-723

40. Pietras RJ, Meadows WR, Fort M, Sharp JT: Hemodynamic alterations in idiopathic myocardiopathy including cineangiography from the left heart chambers. Am J Cardiol 1965, 16: 672-678

41. Johnson RA, Palacios I: Dilated cardiomyopathies of the adult. N Engl J Med 1982, 307: 1051-1058, 1119-1126

42. Takatsu T: Idiopathic cardiomyopathy. Nippon Naika Gakkai Zasshi (J Jpn Soc Int Med) 1977, 66: 1669-1687 (in Japanese)

43. Hamby RI, Catangay P, Apiado O, Kahn AH: Primary myocardial disease: clinical hemodynamic and angiocardiographic correlates in fifty patients. Am J Cardiol 1970, 25: 625-634

44. Fowler NO, Gueron M, Rowlands DT: Primary myocardial disease. Circulation 1961, 23: 498-508

45. Iijima T, Doi M, Kamma H, Horiguchi H, Yazawa T, Ogata T: Pulmonary vasculature in idiopathic dilated cardiomyopathy: a morphometric study. Acta Pathol Jpn 1993, 43: 28-35

46. Romeo F, Pelliccia F, Cianfrocca C, Gallo P, Barilla F, Cristofani $\mathrm{R}$, Reale A: Determinants of end-stage idiopathic dilated cardiomyopathy: a multivariate analysis of 104 patients. Clin Cardiol 1989, 12: 387-392
47. Yamaguchi H, Kaku H, Onodera T, Kurokawa R, Morisada M: Experimental study on the hyperplasia of juxtaglomerular cells under a low-dose, long-term administration of calcium chelating agents: a similar morphological manifestation of Bartter's syndrome. Exp Toxicol Pathol (in press)

48. Trygstad CW, Mangos JA, Bloodworth JM Jr, Lobeck CC: A sibship with Bartter's syndrome: failure of total adrenalectomy to correct the potassium wasting. Pediatrics 1969, 44: 234-242

49. Arant BS, Brackett NC Jr, Young RB, Still WJ: Case studies of siblings with juxtaglomerular hyperplasia and secondary aldosteronism associated with severe azotemia and renal ricketsBartter's syndrome or disease?. Pediatrics 1970, 46: 344-361

50. Taugner R, Waldherr R, Seyberth HW, Erdos EG, Menard J, Schneider D: The juxtaglomerular apparatus in Bartter's syndrome and related tubulopathies. An immunocytochemical and electron microscopic study. Virchows Arch A Pathol Anat Histopathol 1988, 412: 459-470

51. Yamaguchi H, Kaku H. Onodera T, Kurokawa R, Morisada M: Peripolar cells in guinea pigs under experimental hyperplasia of juxtaglomerular cells induced under long-term, low-calcium condition. Exp Toxicol Pathol (in press)

52. Yamaguchi H, Sakaguchi H, Morisada M: Renal lesions following administration of calcitonin. A consideration on the morphological similarities to IgA nephropathy. Exp Pathol 1987, 31: $17-24$

53. Yamaguchi $\mathbf{H}$, Morisada $M$, Sakaguchi $H$ : Renal lesions in hypocalcemic conditions. Phenotypic manifestations of genetical background and renal expressions. Exp Pathol 1991, 41: 10-20

54. Dixon FJ, Vazquez JJ, Weigle WO, Cochrane CG: Pathogenesis of serum sickness. Arch Pathol 1958, 65: 18-28

55. Jones DB: Inflammation and repair of the glomerulus. Am $\mathbf{J}$ Pathol 1951, 27: 991-1009

56. Shibata S, Nagasawa T, Miura K: Nephritogenoside, the receptor glycoprotein for concanavalin $\mathrm{A}$ in rat glomerular basement membrane. Demonstration of alpha-D-glycopyranosyl unit at the non-reducing terminus. Biochim Biophys Acta 1977, 499: $392-403$

57. Shibata S, Miura K: A third glycopeptide (nephritogenoside) isolated from the glomerular basement membrane. J Biochem 1981, 89: $1737-1749$ 